\title{
ОБ АЛГОРИТМАХ АДАПТАЦИИ МОДЕЛИ ОБЪЕКТОВ СО СЛУЧАЙНЫМ ДРЕЙФОМ ПАРАМЕТРОВ. СРАВНИТЕЛЬНЫЙ АНАЛИЗ
}

Изменение свойств многих промышленных объектов носит, как правило, случайный характер. При разработке автоматизированных систем управления ими важен разумный выбор алгоритма адаптации модели. В настоящей статье исследуются свойства оценок дрейфующих параметров объекта в целях сравнения предложенных в литературе алгоритмов.

\section{1. Постановка задачи}

Пусть объект описывается в каждый момент дискретного времени $t=0,1, \ldots, n, \ldots$ линейным по параметрам уравнением

$$
v_{n}=z_{n}^{\mathrm{T}} \beta_{n},
$$

где $v_{n}, z_{n}, \beta_{n}$ - выход, векторы входа и параметров объекта в момент времени $n$ соответственно. Последовательность $\left\{\beta_{n}\right\}$ рассматривается как обобщенно-стационарный временной ряд с независимыми координатами, описываемый параметрической моделью Бокса-Дженкинса $\left[{ }^{1}\right]$.

Изучим свойства оценок известных методов адаптации модели, вычисляемых по наблюдениям входов $z_{n}$ и выхода $y_{n}$, измеренного с аддитивной центрированной ошибкой $h_{n}$, имеющей дисперсию $r$ :

$$
y_{n}=v_{n}+h_{n} .
$$

Величины $\beta_{n}$ и $h_{n}$ принимаем попарно-независимыми.

Для оценивания параметров нестационарных объектов в литературе предлагаются алгоритмы двух типов. У первых отсутствует четкая конкретизация характера нестационарностей объекта. Такие алгоритмы имеют структуру вида

$$
{ }^{*} b_{n}={ }^{*} b_{n-1}+{ }^{*} \Gamma_{n} z_{n}\left(y_{n}-z_{n}^{\mathrm{T}}{ }^{*} b_{n-1}\right) \text {. }
$$

Для шага коррекции ${ }^{*} \Gamma_{n}$ оценок ${ }^{*} b_{n-1}$ в $\left[{ }^{2-4}\right]$ используются, например, следующие выражения:

$$
\begin{aligned}
& { }^{*} \Gamma_{n}=Q^{-1}\left[{ }^{*} \Gamma_{n}-\left(\varrho-z_{n}^{\mathrm{T}}{ }^{*} \Gamma_{n-1} z_{n}\right)^{-1 *} \Gamma_{n-1} z_{n} z^{\mathrm{T}}{ }_{n}^{*} \Gamma_{n-1}\right], \\
& { }^{*} \Gamma_{n}=\left(\omega+z_{n}^{\mathrm{T}} z_{n}\right)^{-1} I, \\
& { }^{*} \Gamma_{n}=\gamma I,
\end{aligned}
$$


где $I$ - единичная матрица; $\varrho, \omega, \gamma$ - заданные постоянные алгоритмов.

Классическими представителями алгоритмов второго типа являются алгоритмы динамической стохастической аппроксимации $\left[{ }^{5}\right]$. Однако в связи с малой степенью конкретизации применительно к задачам оценивания параметров объекта со случайными нестационарностями они нами не рассматриваются.

$\mathrm{B}\left[{ }^{6}\right]$ для оценивания случайно дрейфующих параметров объекта был предложен алгоритм вида

$$
\begin{gathered}
b_{n}=b_{n-1}(1)+\Gamma_{n} z_{n}\left[y_{n}-z_{n}^{\mathrm{T}} b_{n-1}(1)\right], \\
b_{n-1}(1)=\sum_{v=1}^{l} \varphi_{v} \widetilde{b}_{n-v}-\sum_{v=1}^{q} \Theta_{v} a_{n-v}+\mu, \\
\Gamma_{n}=\left(r+z_{n}^{\mathrm{T}} K_{n} z_{n}\right)^{-1} K_{n}, \quad K_{n}=D+D_{n}^{\mathrm{oT}}+D_{n}^{\mathrm{o \sigma}},
\end{gathered}
$$

где $b_{n}$ - текущая оценка параметров $\beta_{n} ; b_{n-1}(1)-$ прогноз $\beta_{n}$; $\sim$ - знак центрирования относительно. безусловного среднего $\mu$ временного ряда $\left\{\beta_{t}\right\} ; a_{n}$ - оценка нормально распределенного белого шума $\alpha_{n}$ с нулевым средним и дисперсией $D ; \Theta_{v}, \varphi_{v}-$ матрицы параметров оператора скользящего среднего порядка $q$ и нестационарного оператора автопегрессии порядка $l$ при сдвиге назад на $v ; D_{n}{ }^{\text {об }}, D_{n}{ }^{\text {от }}-$ дисперсия ошибок прогноза от обучения и отслеживания соответственно (формулы для их определения см. в [ $\left.{ }^{6}\right]$ ).

Ставится задача исследования основных свойств оценок, вычисляемых с помошью алгоритмов (3) и (7), с целью их сравнения на объекте (1). При этом критерием оптимальности служит минимальность смещения и ковариации текущих оценок параметров $b_{n},{ }^{*} b_{n}$, неувязок по модели предсказанного и действительного выхода $e_{n}=v_{n}-z_{n}{ }^{\mathrm{T}} b_{n}$, ${ }^{*} e_{n}=v_{n}-z_{n}{ }^{\mathrm{T} *} b_{n}$ объекта. В компромиссных ситуациях для принятия однозначного решения берется единый критерий - минимальность квадрата математического ожидания неувязок $M\left\{e_{n}^{2}\right\}, M\left\{e_{n}^{2}\right\}$. Критерии нестатистического характера в сравнении не участвуют.

\section{2. Сравнение алгоритмов}

Основные свойства текущих оценок изложены в Приложении. Результаты исследования позволяют сделать следующие выводы:

1. Несмещенные условные оценки дрейфующих параметров и выхода объекта дает алгоритм (7), а смещенные - альтернативный алгоритм (3). Смещение оценок в последнем случае порождается неучетом вероятностных закономерностей дрейфа. Для избежания смещения необходимы некоторые специфические условия, а именно совпадение прогноза по предыстории текущих значений параметров $\boldsymbol{\beta}_{n-1}(1)$ с дрейфом на предыдущем такте $\beta_{n-1}$. Это соблюдается, если дрейф имеет характер случайного блуждания или если возмущения объекта обладают большой инерцией. В этих случаях все методы равноценны в смысле отсутствия смещения. Данный результат действителен в силу того, что ограничения, накладываемые на ширину спектра, более существенны при алгоритмах (3) и менее существенны при алгоритмах (7). Единственным ограничением в (7) является описание дрейфа с помощью обратимой и стационарной модели временного ряда. Надо полагать, что благодаря последнему обстоятельству алгоритм (7), обеспечивающий адап- 
тацию текущих характеристик объектов с быстро изменяющимися свойствами, найдет широкое применение.

2. Шаг уточнения прогноза дрейфующих параметров в (7) минимизирует дисперсию неувязок по модели предсказанного и реального выхода объекта, обусловливая тем самым оптимальное взвешивание значимости текущих наблюдений и априорной информации (процесс накопления и забывания информации). Другие методы позволяют это сделать лишь приблизительно.

3. Условные оценки рассматриваемых алгоритмов несостоятельны в традиционном смысле, хотя и имеют ограниченную дисперсию. Причина этого кроется в скромных возможностях накопления информации об изменяющихся характеристиках объекта.

Для практического применения этих алгоритмов важно учитывать некоторые дополнительные аспекты. К недостаткам алгоритма (7) относятся его сложность и большое количество в априоре определяемых параметров. С этой точки зрения более предпочтительны алгоритмы (3), хотя они ведут к потере точности отслеживания.

Сравнение алгоритмов можно провести и в условиях нарушения принятых предпосылок их вывода. В случае ошибок измерений независимых переменных байесовский алгоритм (7), учитывающий априорную информацию в бо́льшей степени, чем малодостоверные наблюдения, имеет преимущество перед алгоритмами метода наименьших квадратов (3). Обратное наблюдается в случае задания неверной модели дрейфа.

Резюмируя, отметим, что для описания широкого класса нестационарных объектов вида (1) в смысле единого критерия $Q$ более предпочтителен алгоритм (7). Однако, когда существует возможность выбора другого критерия качества (нестатистического характера) или дрейфа с иной спецификой, полезными могут оказаться и другие алгоритмы.

\section{ПРИЛОЖЕНИЕ}

\section{Основные свойства оценок}

Качество текущих оценок ${ }^{*} b_{n}, b_{n}$ определяется точностью измерения выхода и отклонением $b_{n-1}$ (или прогноза $\left.b_{n-1}(1)\right)$ от параметров $\beta_{n}$. Последнее выражается в виде

$$
\begin{gathered}
{ }^{*} b_{n-1}-\beta_{n}=\left[{ }^{*} b_{n-1}-\beta_{n-1}\right]+\left[\beta_{n-1}-\beta_{n-1}(1)\right]+ \\
+\left[\beta_{n-1}(1)-\beta_{n}\right]={ }^{*} \Delta_{n-1}+\Delta_{n}{ }^{\mathrm{cp}}+\alpha_{n}
\end{gathered}
$$

где ${ }^{*} \Delta_{n-1}$ - отклонение оценок ${ }^{*} b_{n-1}$ от $\beta_{n-1} ; \Delta_{n}{ }^{\mathrm{cp}}-$ разница между параметром $\beta_{n-1}$ и прогнозом с момента $n-1$ на один шаг внеред $\beta_{n-1}(1)$.

Согласно результатам [ $\left.{ }^{6}\right]$, справедливо

$$
b_{n-1}(1)-\beta_{n}=\Delta_{n}^{\text {об }}+\Delta_{n}^{\text {от }}+\alpha_{n}
$$

где $\Delta_{n}$ об - ошибка прогноза, порождаеммая неопределенностью начальных условий, в разностном уравнении алгоритма $(7) ; \Delta_{n}{ }^{\text {от }}$ - ошибка прогноза, порождаемая неточным отслеживанием дрейфа на предыдущих тактах. Ошибки $\Delta_{n}{ }^{\text {об }}$ и $\Delta_{n}{ }^{\text {от }}$ имеют нулевое математическое ожидание и дисперсию $D_{n}{ }^{\text {об }}, D_{n}{ }^{\text {от }}$.

Согласно постановке задачи, результатам $\left[{ }^{6}\right]$ и определению (П. 1), 
величины $h_{n}, \alpha_{n},{ }^{*} \Delta_{n-1}$ (или $\Delta_{n}{ }^{\circ \sigma}, \Delta_{n}{ }^{\text {от) }}$ ) попарно-независимы. В момент $n-1$ они неизвестны и поэтому рассматриваются как случайные.

1. С м е щ н и е о ц ен ок $b_{n},{ }^{*} b_{n}, e_{n},{ }^{*} e_{n}$. Используя выражения (1), (2) и алгоритм (7), получаем

a) $M\left\{\Delta_{n}\right\}=M\left\{b_{n}-\beta_{n}\right\}=$

$$
=M\left\{b_{n-1}(1)-\beta_{n}\right\}+\Gamma_{n} z_{n}\left[M\left\{h_{n}\right\}-z_{n}^{\mathrm{T}} M\left\{b_{n-1}(1)-\beta_{n}\right\}\right]=0,
$$

так как

$$
M\left\{b_{n-1}(1)-\beta_{n}\right\}=M\left\{\Delta_{n}^{\text {об }}\right\}+M\left\{\Delta_{n}^{\text {оT }}\right\}+M\left\{\alpha_{n}\right\}=0 .
$$

$$
M\left\{e_{n}\right\}=z_{n}^{\mathrm{T}} M\left\{\Delta_{n}\right\}=0 .
$$

То же самое при алгоритмах (3):

в) $M\left\{{ }^{*} \Delta_{n}\right\}=M\left\{{ }^{*} b_{n}-\beta_{n}\right\}={ }^{*} \Gamma_{n} z_{n} M\left\{h_{n}\right\}+\left[I-{ }^{*} \Gamma_{n} z_{n} z_{n}\right] M\left\{{ }^{*} b_{n-1}-\beta_{n}\right\}=$

$$
=\left[I-{ }^{*} \Gamma_{n} z_{n} z_{n}^{\mathrm{T}}\right]\left[M\left\{{ }^{*} \Delta_{n-1}\right\}+\Delta_{n}^{\mathrm{cp}}\right]=
$$

$$
\begin{gathered}
=\left(I-{ }^{*} \Gamma_{n} z_{n} z_{n}^{\mathrm{T}}\right)\left[\Delta_{n}^{\mathrm{cp}}+\left(I-{ }^{*} \Gamma_{n-1} z_{n-1} z_{n-1}^{\mathrm{T}}\right)\left[\Delta_{n-1}^{\mathrm{cp}}+\ldots .\right.\right. \\
\left.\left.+\left(I-{ }^{*} \Gamma_{0} z_{0} z_{0}^{\mathrm{T}}\right) \Delta_{0}^{\mathrm{cp}}\right] \ldots\right],
\end{gathered}
$$

так как

$$
M\left\{{ }^{*} b_{n-1}-\beta_{n}\right\}=M\left\{{ }^{*} \Delta_{n-1}\right\}+\Delta_{n}^{\mathrm{cp}} .
$$

Здесь предполагается, что априорная оценка $b_{-1}$ несмещенная:

$$
M\left\{b_{-1}-\beta_{-1}\right\}=0 .
$$

г)

$$
M\left\{{ }^{*} e_{n}\right\}=z_{n}^{\mathrm{T}} M\left\{{ }^{*} \Delta_{n}\right\} \text {. }
$$

В отличие от оценок ${ }^{*} b_{n},{ }^{*} e_{n}$, оценки $b_{n}, e_{n}$ несмещенные. Следовательно, в общем случае справедливо

$$
\left|M\left\{{ }^{*} b_{n}-\beta_{n}\right\}\right| \geqslant M\left\{b_{n}-\beta_{n}\right\}=0, \quad M\left\{{ }^{*} e_{n}\right\} \geqslant M\left\{e_{n}\right\}=0 .
$$

2. К о в а ри ация оце нок $b_{n},{ }^{*} b_{n}, e_{n},{ }^{*} e_{n}$. Нетрудно показать, что соблюдается равенство

a) $D_{n}=M\left\{\left(b_{n}-\beta_{n}\right)\left(b_{n}-\beta_{n}\right)^{\mathrm{T}}\right\}=$

$$
=\Gamma_{n} z_{n} z_{n}^{\mathrm{T}} \Gamma_{n}^{\mathrm{T}}+\left[I-\Gamma_{n} z_{n} z_{n}^{\mathrm{T}}\right] K_{n}\left[I-\Gamma_{n} z_{n} z_{n}^{\mathrm{T}}\right]^{\mathrm{T}}=r \Gamma_{n}
$$

поскольку

$$
\begin{gathered}
K_{n}=M\left\{\left(b_{n-1}(1)-\beta_{n}\right)\left(b_{n-1}(1)-\beta_{n}\right)^{\mathrm{T}}\right\}= \\
=M\left\{\alpha_{n} \alpha_{n}^{\mathrm{T}}\right\}+M\left\{\left(\Delta_{n}^{\mathrm{oT}}\right)\left(\Delta_{n}^{\mathrm{oT}}\right)^{\mathrm{T}}\right\}+M\left\{\left(\Delta_{n}^{\mathrm{o \sigma}}\right)\left(\Delta_{n}^{\mathrm{o \sigma}}\right)^{\mathrm{T}}\right\}=D+D_{n}^{\mathrm{oT}}+D_{n}^{\circ} .
\end{gathered}
$$

$$
M\left\{e_{n}^{2}\right\}=z_{n}^{\mathrm{T}} D_{n} z_{n}
$$

Оценки ${ }^{*} b_{n},{ }^{*} e_{n}$ оказались смещенными. Поэтому рассеяние оценок выражается сначала в) и г) относительно смещенных средних значений, а затем д) и е) относительно действительных $\beta_{n}, 0$.

в) ${ }^{*} D_{n}^{\mathrm{cM}}=M\left\{\left({ }^{*} b_{n}-M\left\{{ }^{*} b_{n}\right\}\right)\left({ }^{*} b_{n}-M\left\{{ }^{*} b_{n}\right\}\right)^{\mathrm{T}}\right\}=$ 


$$
={ }^{*} \Gamma_{n} z_{n} r z_{n}{ }^{*} \Gamma_{n}^{\mathrm{T}}+\left[I-{ }^{*} \Gamma_{n} z_{n} z_{n}^{\mathrm{T}}\right]^{*} K_{n}^{\mathrm{cM}}\left[I-{ }^{*} \Gamma_{n} z_{n} z_{n}^{\mathrm{T}}\right]^{\mathrm{T}},
$$

где

$$
{ }^{*} K_{n}^{\mathrm{cm}}=M\left\{\left({ }^{*} b_{n-1}-\beta_{n}\right)\left({ }^{*} b_{n-1}-\beta_{n}\right)^{\mathrm{T}}\right\}=D+{ }^{*} D_{n-1}^{\mathrm{cm}} \text {. }
$$

г)

$$
M\left\{\left({ }^{*} e_{n}-M\left\{{ }^{*} e_{n}\right\}\right)^{2}\right\}=z_{n}^{\mathrm{T}}{ }^{*} D_{n}^{\mathrm{cM}} z_{n} .
$$

д) ${ }^{*} D_{n}=M\left\{\left({ }^{*} b_{n}-\beta_{n}\right)\left({ }^{*} b_{n}-\beta_{n}\right)^{\mathrm{T}}\right\}={ }^{*} D_{n}^{\mathrm{cM}}+M\left\{{ }^{*} \Delta_{n}\right\} M^{\mathrm{T}}\left\{{ }^{*} \Delta_{n}\right\}$.

e)

$$
M\left\{{ }^{*} e_{n}^{2}\right\}=z_{n}^{T^{*}} D_{n} z_{n} .
$$

Выражение шага $\Gamma_{n}$ алгоритма (3) минимизирует ковариацию неувязок (П. 9) и поэтому является оптимальным. Любая из формул расчета шага (4)-(6) не обеспечивает минимум ковариации (П. 11) или единого критерия (П. 13). Очевидно, соблюдаются неравенства

$$
{ }^{*} D_{n}>D_{n}, \quad M\left\{{ }^{*} e_{n}^{2}\right\}>M\left\{e_{n}^{2}\right\}
$$

3. Состоятельность оценок $b_{n},{ }^{*} b_{n}$.
a) $\lim _{t \rightarrow \infty} D_{t} \neq 0$,
если $r \neq 0$ или $D \neq 0$.

б) $\lim _{t \rightarrow \infty} * D_{t} \neq 0$

если $r \neq 0$ или $D \neq 0$.

В случае случайного дрейфа параметров объекта оценки $b_{n},{ }^{*} b_{n}$ несостоятельны.

\section{ЛИТЕРА Т У Р А}

1. Бокс Дж., Д женкинс Г. Анализ временных рядов. Прогноз и управление. М., 1974.

2. Бородюк В. П., Л ецки й Э. К. Статистическое описание промышленных объектов. М., 1971.

3. Р ай бм а Н Н. С., Ч а де е в В. М. Адаптивные модели в системах управления. M., 1966.

4. Л ецкий Э. К. Адаптивная система слежения за изменяющимися параметрами характеристики функционального преобразователя. - Автоматика и телемеханика, 1970, № 2, с. 43-49.

5. Цыпкин Я. 3., Каплин ский А. И., Л аринов К. А. Алгоритмы адаптации и обучения в нестационарных условиях. - Техническая кибернетика, 1970, № 5 , с. $9-21$.

6. Тенн о Р. Идентификация объектов со случайным дрейфом параметров. - Изв. АН ЭССР, Физ. Матем., 1978, т. 27, № 2, с. 159-167.

Научно-исследовательский

и проектно-технологический институт

Таллинского электротехнического завода им. М. Й. Калинина
Поступила в редакцию 22/VI 1977 
R. TENNO

\section{JUHUSLIKULT MUUTUVATE PARAMEETRITEGA \\ OBJEKTIDE ADAPTEERIMISE ALGORITMIDEST. VORDLEV ANALUUS}

Artiklis on võrreldud seni teadaolevaid mittestatsionaarsete objektide parameetrite jooksva hindamise meetodeid sisend-väljundandmete alusel ja saadud objekti juhuslikult muutuvaid parameetreid järgivate hinnangute nihke ja kovariatsiooni avaldised. On esitatud vaadeldavat klassi objektide korral parim mudeli adapteerimise algoritm.

R. TENNO

\section{A COMPARATIVE ANALYSIS OF ALGORITHMS FOR ADAPTION MODELS OF PLANTS WITH STOCHASTICALLY VARYING PARAMETERS}

This paper compares known methods for the identification of non-stationary plant parameters from input-output data. Expressions for bias and variance of components of the errors in tracking stochastically varying parameters of the plant are obtained. The best aigorithm is proposed for the adaption of a model of a considerable cliss of plants. 